\title{
Distributed Software Architecture of PEBB-based Plug and Play Power Electronics Systems
}

\author{
Jinghong Guo, Ivan Celanovic, and Dushan Borojevic \\ Center for Power Electronics Systems \\ The Bradley Department of Electrical and Computer Engineering \\ Virginia Polytechnic Institute and State University \\ Blacksburg, VA 24061-0179
}

\begin{abstract}
A novel hierarchical software architecture, based on PEBB concept, is proposed to build flexible, configurable and reusable power electronics system control software. Some important issues about software design will be addressed. And as an example, a three-phase DC-AC inverter will be implemented under this hierarchical architecture.
\end{abstract}

\section{INTRODUCTION}

In traditional centralized digitally controlled power electronics systems, construction, debugging and maintenance of the control software are complicated and difficult because of the lack of standardization and modularization, and also because of the strong dependence on system hardware. In computer research, there have been many mature technologies addressing similar issues. For example, an operating system [1] manages system resources, and handles the interfaces to all kinds of peripherals. The object-oriented technology [2] provides a successful way to both reduce software design complexity and modularize software. Plug and Play (PnP) [3] technology gives a computer system the capability of automatic configuration, so that the system construction becomes much user-friendly.

The concept of power electronics building blocks (PEBB) [4] provides a way to hardware standardization of power electronics systems. Based on PEBB concept, this paper proposes to apply some computer software concepts and technologies to design flexible, reusable, automatically configurable power electronics system control software.

To do this, the control software will be functionally divided into hierarchical levels. By building modularized software objects within each level, standardizing interfaces between levels, the application software will be independent of the hardware specifications of power stage. As long as supporting the standardized interfaces between levels, products from different vendors can communicate and work with each other. Furthermore, if both sides of an interface support device self-identification and system resources assignment, then the so-called PnP can be implemented at this interface.

Section II will discuss the hierarchical division of control software of power electronics systems, functionality of each

This work was supported primarily by the ERC. Program of the National Science Foundation under Award Number EEC-9731677. level, and how to draw boundaries between levels. Section III will address software modularization issues by applying object-oriented technology. In section IV, other important software issues surrounding implementation of reliable power electronics control software will be discussed. Section V will concentrate on the software design at the middle level of the whole hierarchical architecture, which implements a converter control algorithm. In Section VI, a three-phase DCAC closed loop inverter is designed as an example to show the implementation of the hierarchical PnP power electronics system control software. Experimental results will be shown.

\section{HiERARChICAL SOFTWARE ARChITECTURE OF PNP POWER ELECTRONICS SYSTEMS}

\section{A. Hierarchical division of power electronics control}

Fig. 1. shows a way to functionally divide the control in power electronics systems. In this hierarchical architecture, the control software is divided into 3 hierarchies-high-level control, application manager (AM) and hardware manager (HM). The division is based on functionality. The high-level control performs tasks at the system level, such as responding to users' commands, coordinating performances between converters, and monitoring system execution, etc. An AM is a controller at the converter level. It calculates control information-for example, PWM commands-for a converter. An HM is a controller at the PEBB level. The HM for a PEBB generates PWM pulses to control switch operations, according to the control information received from the AM, monitors the status of a PEBB, and performs fast protection within a PEBB.

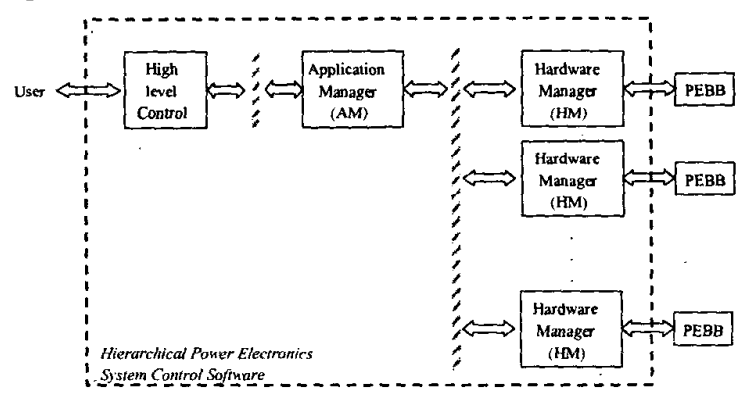

Fig. 1. Plug and Play power electronics system architecture. 
The purpose of drawing boundaries between hierarchical levels is to make each level as functionally self-contained as possible, and make higher level application software as independent of lower level hardware as possible. Next, we will take AM-HM levels as an example to show how to draw boundaries between levels.

\section{$B$. Functional division between $A M$ and $H M$}

For AM-HM levels, the control algorithm of a converter is application specific, and generating pulses for switches is directly dependent on features of the power stage hardware. Roughly, the software division between AM and HM can be drawn in such a way that the AM level implements the converter control algorithm, the HM level generates switch pulses, and the interface of AM-HM levels translates PWM commands into switch PWM pulse related information.

However, how to draw the software boundary between AM - HM levels accurately, for example, where a modulator should be implemented, has more than one solution. The boundary drawing can be arbitrary, which means functions implemented at each level and data transferred between hierarchies are well defined, no matter what kind of hardware is used at each level. A better solution allows boundaries float somewhat between different systems and applications, so that higher system flexibility can be achieved.

For example, if the HM has enough calculation capability, some calculation can be shifted to the HM level. Thus the workload of the AM can be reduced, while the HM can be more efficiently used. On the contrary, if the HM is as simple as a logical circuit of some data buffers and timers, the AM should take over the calculation work as much as possible. The data transferred through each interface will vary with the floating of software boundaries. If the boundary floating is achieved by software instead of hardware, this method of interface definition will make the system structure more flexible and open without additional hardware requirement.

\section{Modularize PoWER EleCtronics CONTROL SOFTWARE BY OBJECT-ORIENTED TECHNOLOGY}

Another important goal of the PEBB-based PnP power electronics systems design is to modularize and standardize power electronics control software, hence make construction, debugging and maintenance of the control software much easier. In computer research, object-oriented technology [5] is a main method to achieve software modularization and reduce software complexity.

Fig. 2. shows the structure of a power electronics software object. Each object has a well-defined interface, a parameter field, inside code and exception handler. The interface indicates the communication with other objects, such as inputs, output and data format. The interface also defines the function that the object provides. The inside code implements the function of the object, with implementation of code invisible to the outside. The parameter field stores parameters that may affect or direct the execution of the object, such as references, threshold values, etc. The purpose of using a parameter field is to make an object execution as selfcontained as possible. The parameters will be set at the control software initialization stage. The execution of an object will depend on its inputs and inside parameters, such that global variables can be avoided. Reducing use of global variants will make debugging and maintenance of software much easier.

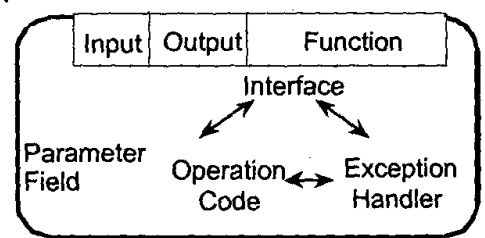

Fig. 2. Structure of power electronics control software object.

Fig. 3. shows an example of a current regulator object. From the outside of the object, what known is this object works as a current regulator, takes $d$ channel current reference, $q$ channel current reference, $d$ channel current and $q$ channel current as input, outputs voltages references for $d$ and $q$ channels. How the regulator is implemented is invisible to the outside.

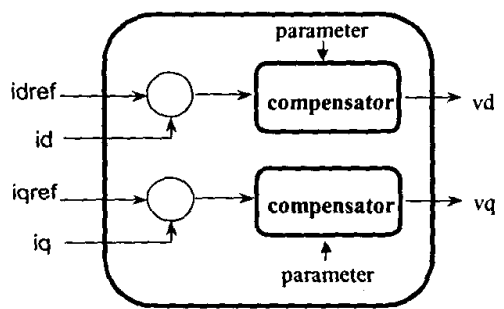

Fig. 3. An object example: current regulator.

Using object-oriented software structure can also benefit the reliability of software, which will be discussed later in section IV.

\section{TIMER WATCH DOG AND EXCEPTION HANDLING MECHANISMS}

Two other important issues about building reliable control software will be discussed in this section: ensuring that all the time constraints can be satisfied, and protecting the system from exceptions.

Power electronics systems are hard real-time systems. The correctness of a result does not only mean that the value of the result is correct, but also means that the result must be achieved before some time deadline. If the AM embedded code is stuck for some unknown reason-for example, the code cannot return from a interrupt service routine (ISR)the AM should also give out some converter control information instead of losing control of the converter. For a critical system performance, a time limit will be set. If the time for this performance expires, and the performance is not finished as expected, then the watch dog timer for the performance will force the performance to end. And some 
reasonable but possibly not accurate results will be given out to keep the converter running.

Object-oriented software offers a method of exception handling at the object level. Each software object has an exception handler to deal with exceptions raised within the object. Only if there is an exception that cannot be handled inside the object, will this exception be propagated to the caller of the object. The purpose is to reduce the effect of an exception within an object to the outside as little as possible.

\section{SOFTWARE STRUCTURE DESIGN OF AM}

So far the hierarchical division of power electronics control software has been discussed. This section presents the software design of AM, which implements control at the converter level.

\section{A. Software structure of $A M$}

Traditionally, converter control means when the converter is running; the controller should generate proper control commands every switching period, make sure that all computations meet their deadlines, and handle certain exceptions. This is the type of control that AM must perform at the running time.

To do PnP at the AM-HM level requires additional functionality at AM. Before the AM can control HMs, the AM must configure the lower level components according to both application requirements and lower level hardware specifications, which is called converter configuration. To support PnP at AM--HM levels, an HM should be able to provide the self-identification information to the $A M$, and accept the system resource assignment from the AM. When the system starts up, the AM should have the ability to get PEBB hardware specifications from $\mathrm{HMs}$, and configure HMs in such a way that the requirements of the specific application can be satisfied. Also the AM should be able to assign system resources, such as addresses and data buffers, to HMs without any conflict.

So besides implementing the converter control algorithm, the AM should also provide some basic system services for $\mathrm{PnP}$ configuration, and handle interfaces to other levels. Normally, these functions are implemented by operating system in computer systems. Similarly, an embedded operating system dedicated for power electronics systems, named power electronics operating system (PEOS) is introduced. The whole control software systems at AM are composed of two subsystems: converter real-time control subsystem and PEOS, as shown in Fig. 4.

The converter real-time control subsystem can be seen as an object, and is application specific. A converter real-time control object is invoked once every switching period. It may have many sub-objects, such as sensor reading, filters, regulators, etc. Different software object combinations implement different applications. While the embedded realtime PEOS provides basic system services, such as system configuration, system resource allocation and maintenance, communication with software objects at other hierarchies, etc. The PEOS can be optimized for different applications. It is the embedded PEOS that makes the converter control algorithm truly independent of the infrastructure of the HM level.

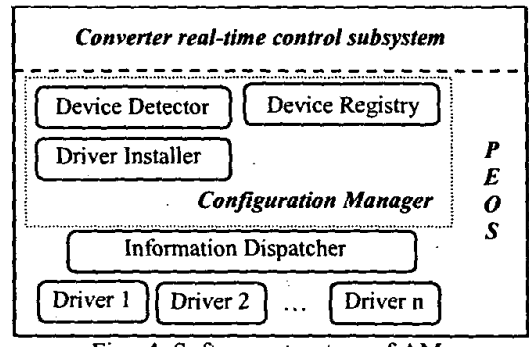

Fig. 4. Software structure of AM.

\section{B. Configuration Manager}

In the PEOS, an object named configuration manager (CM) handles the converter PnP configuration. As shown in Fig. 4., the CM is composed of three components: the device detector, the driver installer and the device registry. The device detector searches the AM-HM network for HM that has not been configured, and assigns unique address to newly detected HM. The driver Installer installs a software driver for a newly detected HM. The device registry is a database, which contains the driver information of all HMs controlled by one AM. The CM has two primary functions: to provide the software interface through which configuration information can be exchanged between the AM and HMs; to coordinate between the device detector, the device installer and the device registry.

\section{Drivers}

To build open communication between levels, the concept of driver will be introduced. A driver, in computer terminology, is defined as a set of functions that manipulates a hardware device [6]. In AM-HM levels, a driver is implemented as software objects, which are used by the AM to communicate with a specific HM.

In a traditional centralized digitally controlled power electronics system, the designer of the application software must know every detail about V/O ports, the data format of the converter controller and power stage interface in order to build up the communication between the controller and the power stage. Even small changes in the hardware setup will cause a lot of modifications in the control software. By using a driver, all the hardware specifications and communication details will be encapsulated within the driver, so that the application can be independent of the infrastructure of other levels,

It has been mentioned in section II that floating boundary definition helps to build flexible and open systems. To support floating boundary, the format of data, instead of the content of data, exchanged between levels should be defined. The data packet format at the AM--HM interface is shown in Fig. 5. In the packet head, Packet_Type indicates the type of 
the data packet; Src_Addr indicates the sender of the packet; Dest Addr holds the destination address of the packet; and Length indicates the length of the packet body, which is variable. Only the software at the two sides of a communication can understand the content of a data packet, i.e. that body of the packet.

$$
\begin{aligned}
& \text { Head } \\
& \begin{array}{|l|l|l|l|}
\hline \text { Packet_Type } & \text { Src_Addr } & \text { Dest_Addr } & \text { Length } \\
\hline
\end{array} \\
& \begin{array}{|l|l|}
\hline \text { Dody } \\
\hline \text { Data byte 1 } & \text { Data byte } 2 \\
\hline
\end{array}
\end{aligned}
$$

Fig. 5. Data packet format at $A M-H M$ interface.

\section{Information Dispatcher}

Normally the results from the converter real-time control are for a whole converter. While each HM needs control information specific for its PEBB. Information Dispatcher is such a software object, which decomposes the converter related control information into control information for each PEBB. Further, a driver translates the control information into the format that can be understood by its corresponding HM. With Information Dispatcher and drivers, the control algorithm can really be independent of the hardware at the PEBB level.

\section{A PEBB-BASED PLUG AND PLAY THREE-PHASE INVERTER DESIGN}

In this section, a three-phase inverter will be presented as an example to show the implementation of the system control software on the software architecture and software technologies discusses above.

\section{A. System architecture}

Fig. 6. shows the system architecture. AM-HM level communication is through PES-Net [7], which is optic fiber serial link, with transmission capacity of $125 \mathrm{Mbps}$.

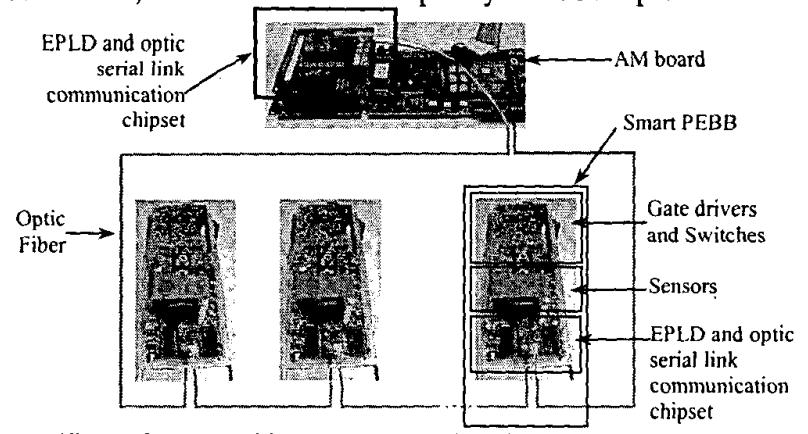

Fig. 6. System architecture of PEBB based PnP DC-AC converter.

\section{B. PnP configuration design}

Fig. 7. shows the $\mathrm{PnP}$ configuration mechanism of the Configuration Manager, based on PES-Net.

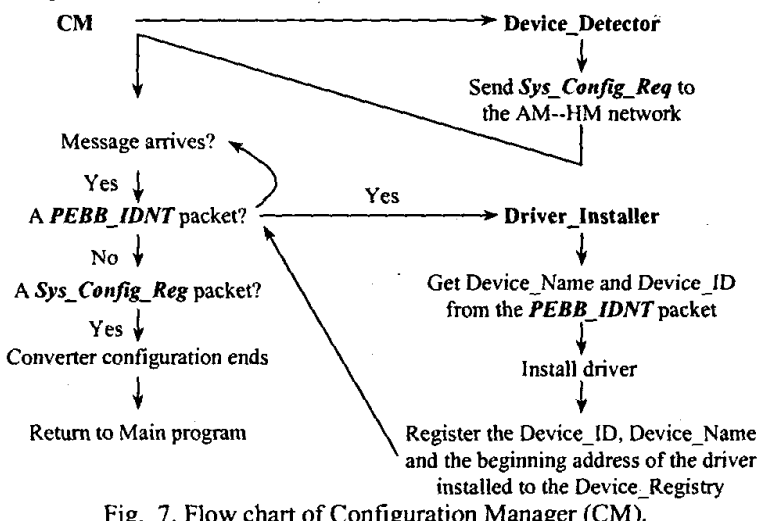

When the converter configuration begins, the device detector broadcasts a system configuration request to the AM-HM network. Fig. 8. shows the data format of a system configuration request packet.

Head

\begin{tabular}{|l|c|c|c|} 
Packet_Type & Src_Addr & Dest_Addr & Length \\
\hline Sys_Config_Req & $000 \ldots 0$ & $111 \ldots 1$ & 1 \\
\hline
\end{tabular}

Body

NewDevice ID

Fig. 8. Data format of Sys_Config_Req packet.

The Packet_Type field indicates that this is a system configuration request. The Src_Addr field is all zeros, which is a special address stands for the AM, because for a converter configuration packet, the sender can only be the AM. The Dest Addr is all ones, another special address, meaning the packet is a broadcast message. Every node in the network can receive this packet. The only field in the packet body is Device ID, which contains the next available device address for a PEBB that has not been configured.

When a HM receives a converter configuration packet, it will check itself first to determine whether it has been configured or not. If the PEBB has been configured, it will simply pass the packet to the next node on the network. If the HM has not been configured, it will send its selfidentification packet back to the AM. The data format of the device self-identification packet is shown in Fig 9. The only information a PEBB tells the AM is its device name. A device name is unique for each type of $P E B B$, which can be used by the AM to find and install a proper driver for the PEBB. 
Head

\begin{tabular}{|c|c|c|c|}
\hline Packet Type & Src_Addr & Dest_Addr & Length \\
\hline Device_IDN & Device_ID & 0000 & 1 \\
\hline
\end{tabular}

Body

$$
\text { Device_Name }
$$

Fig. 9. Data format of Device_IDN packet

When the AM receives a device self-identification packet, the device installer will install a driver for the PEBB according to the device name it gets from the packet. And the device installation information will be logged in the device registry. The device registry is a kind of system resource and is maintained by the PEOS. It tells the converter control the code location of the driver for a specific PEBB. Each device has an entry in device registry. And each entry has three fields, as shown in TABLE 1.

TABLE 1

DEVICE_REgISTRY ENTRY

\begin{tabular}{|l|l|}
\hline Item Name & Description \\
\hline Device_ID & $\begin{array}{l}\text { Identification of the PEBB in the } \\
\text { AM-PEBB network }\end{array}$ \\
\hline Device_Name & Name of the device \\
\hline Driver_Entry & $\begin{array}{l}\text { The entry address of the driver in the } \\
\text { memory }\end{array}$ \\
\hline
\end{tabular}

When the $\mathrm{AM}$ receives a system configuration request packet, it knows that all the devices on the AM-HM network have been configured. This also implies that the system configuration is finished.

\section{Converter control algorithm design}

For this three-phase DC-AC PnP inverter application, the current loop is closed. Fig. 10. shows the inverter control algorithm implemented by software objects.
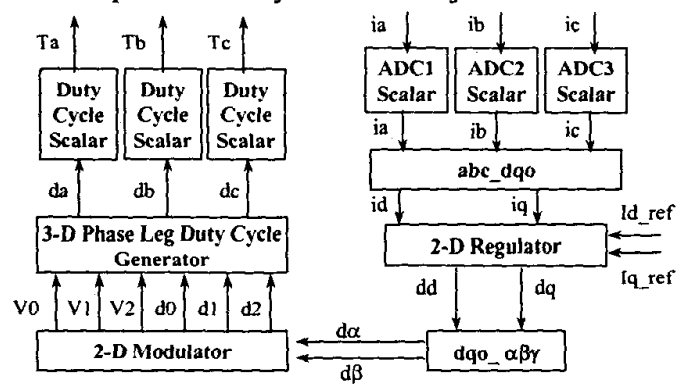

Fig. 10. Closed loop inverter control algorithm implemented by software objects.

A "ADC Scalar" object takes sensor values as input and outputs value after scaling and offsetting. Object "abc_dqo" transforms abc coordinates to dqo coordinates. Object "3-D phase Leg Duty Cycle Generator" is the information dispatcher. And Object "Duty Cycle Scalar" transform duty cycle into clock ticks, which can be used directly by a specific HM to generate switching pulses.

For the exception handling, before the duty cycle value is output to HM driver, the validity of the value will be checked. If the duty cycle is beyond the valid range, for example, outside $[0,1]$, the stored previous calculation result will be used as the output.

\section{E. Experimental results}

The IGBT devices used in the smart PEBB are POWEREX, 1200V/300A. Fig. 11. shows the experimental waveforms under conditions:

1) DC input voltage: $200 \mathrm{~V}$;

2) Switching frequency: $20 \mathrm{kHz}$;

3) Current loop closed;

4) Inductive load: $\mathrm{R}=3 \mathrm{ohm}, \mathrm{L}=0.3 \mathrm{mH}$.

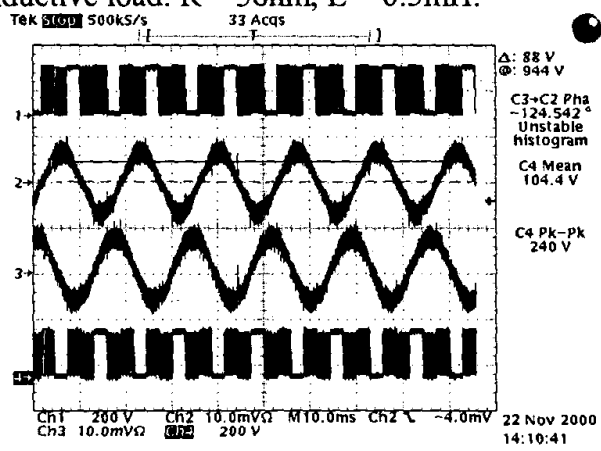

Fig. 11. Phase current and voltage waveforms.

In Fig. 11, Ch1 shows the voltage of phase $\mathrm{A}$, with $200 \mathrm{~V} /$ div; $\mathrm{Ch} 2$ shows the phase current of phase $\mathrm{A}$, with $5 \mathrm{~A} / \mathrm{div}$; $\mathrm{Ch} 3$ and $\mathrm{Ch} 4$ shows the voltage and current of phase $\mathrm{B}$ respectively.

\section{CONCLUSION AND FUTURE WORK}

This paper presents a novel hierarchical software structure of PEBB-based $\mathrm{PnP}$ power electronics systems. In the hierarchical structure, the power electronics system control software is divided into three levels-high-level control, application manager and hardware manager. Software design issues, such as boundary definition, software modularization, exception handling, are addressed. Software design of AM is discussed in detail. A three-phase DC-AC PEBB-based PnP inverter is implemented as an example to show the implementation of the AM.

Future work includes investigating more suitable software architecture for control distribution, concurrent execution and software reuse. Dataflow is a promising candidate. Also more flexible control partition method needs to look into.

\section{REFERENCES}

[1] S. Haisler, "The design of operating systems for small computer system," A Wiley_Interscience Publication, 1983, pp. 18-26. 
[2] G. Blair, J. Gallagher, D. Hutchison and D. Shepherd, "Object-oriented languages, systems and applications," $A n$ Imprint of John Wiley \&Sons, 1991, pp.1-3.

[3] J. Kelsey, "Programming Plug and Play," by Sams Publishing, 1995, pp. 2.

[4] T. Ericsen and A. Tucker, "Power electronics building blocks and potential power modulator applications," IEEE Conference Record of the 23rd International Power Modulator Symposium, New York, NY, pp. p.12-15; 1998.

[5] G. Booch, "Object oriented design", by the Benjamin/Cummings Publishing Company, 1991, pp.32-37.

[6] K. Hazzah, "Writing windows VxDs and device drivers", [5] Karen Hazzah, "Writing windows VxDs and device drivers," by R\&D books, 1997, pp.1.

[7] I. Milosavljevic, D. Borojevic., I. Celanovic, "Modularized Communication and Control Structure for Power Converters," $8^{\text {th }}$ European . Conference on Power Electronics and Applications, EPE, September 1999. 